\title{
Beta-bloqueantes en cirugía no cardíaca: evidencia promisoria pero insuficiente
}

\section{Objetivo}

Valorar el efecto de los Betabloqueantes (BB) perioperatorios en cirugía no cardíaca.

\section{Fuentes de Datos}

Se exploró MEDLINE, EMBASE, SciSearch, búsqueda manual revistas de anestesia, expertos, referencias de los estudios clave.

\section{Selección de estudios}

Dos investigadores hicieron independientemente la selección de Ensayos Clínicos Controlados Aleatorizados (ECCA) que evaluaran el efecto de los BB dentro de los 30 días de una cirugía no cardíaca para los siguientes resultados: muerte, muerte cardíaca, infarto de miocardio (IM) fatal y no fatal, insuficiencia cardíaca, broncoespasmo y bradicardia o hipotensión que requiriesen tratamiento. Los desacuerdos se resolvieron por consenso.

\section{Extracción de datos}

Dos investigadores extrajeron independientemente los datos de resultados y criterios de calidad (como enmascaramiento de la asignación*, interrupción temprana y ceguera de pacientes, médicos tratantes, encargados de recolectar la información y evaluadores). Los desacuerdos se resolvieron por consenso.

\section{Resultados principales}

Se incluyeron 22 ECCA (2437 pacientes) de 4964 estudios identificados. El seguimiento sólo se limitó hasta el final de la cirugía o salida de la recuperación en seis ECCA. Cinco ECCA no cumplieron con alguno de los criterios de calidad. Los BB perioperatorios (análisis por intención de tratar*) sólo redujeron el resultado combinado de eventos cardiovasculares (CV) mayores, pero además de una importante heterogeneidad ${ }^{*}$, dado el bajo número de eventos (18 muertes cardiovasculares, $58 \mathrm{IM}$ no fatales y 7 paros cardíacos no fatales) perdía significancia estadística con un IC99\%*. Los BB no mostraron ningún efecto beneficioso para los resultados individuales pero sí aumentaron la bradicardia o hipotensión que requirió tratamiento (Tabla). Mientras tres ECCA que fallaban en los criterios de calidad (Poldermans, Zaug y Urban) tuvieron una marcada reducción de Eventos CV mayores de 87\% (IC95\% 62-96\%), en los cinco ECCA restantes la RRR sólo mostró una tendencia no significativa del $18 \%$.

\begin{tabular}{|c|c|c|c|c|c|c|}
\hline Resultados & BB $(n / N)$ & Control $(n / \mathbb{N})$ & $\mathbf{R R}$ & (IC 95\%) & (IC 99\%) & $\mathrm{I}^{2} @$ \\
\hline Muerte & 9/453 & $19 / 454$ & 0,56 & $0,14-2,31$ & $0,09-3,60$ & $57 \%$ \\
\hline Muerte cardiovascular & $5 / 453$ & $13 / 454$ & 0,40 & $0,14-1,15$ & $0,10-1,60$ & $0 \%$ \\
\hline IM no fatal & $21 / 441$ & $37 / 412$ & 0,38 & $0,11-1,29$ & $0,08-1,88$ & $45 \%$ \\
\hline Eventos CV mayores" & $28 / 589$ & $55 / 563$ & 0,44 & $0,20-0,97$ & $0,16-1,24$ & $42 \%$ \\
\hline Hipotensión clínica & $216 / 986$ & $147 / 726$ & 1,27 & $1,04-1,56$ & $0,97-1,66$ & $6 \%$ \\
\hline adicardia clínica & $87 / 621$ & $31 / 575$ & 2,27 & $1,53-3,36$ & $1,36-3,80$ & $3 \%$ \\
\hline
\end{tabular}

\#Eventos cardiovasculares mayores: muerte cardiovascular, IM no fatal y paro cardíaco no fatal.@I2: \% de heterogeneidad entre estudios más allá del azar $(\mathrm{I} 2<25 \%$ sugiere baja $\mathrm{y}>75 \%$ alta heterogeneidad).

\section{Conclusiones}

La evidencia de reducción de eventos cardiovasculares mediante los BB perioperatorios es alentadora pero insuficiente como para establecer conclusiones definitivas

Fuente de financiamiento: Instituto Canadiense de Investigación en Salud.

\section{Comentario}

Anualmente de 500.000 a 900.000 adultos en todo el mundo son sometidos a cirugías no cardiacas y de $1-5 \%$ sufren eventos cardiovasculares relacionados que son de gran impacto en la salud pública'. Varias guías han recomendado el uso de BB perioperatorios, sin embargo al momento sólo 83 eventos cardiovasculares registrados en los ECCA podrían avalarlas. Luego que el Colegio Americano de Médicos aprobara la guía de cuidados cardíacos perioperatorios, sus dos autores insertaron sus propias recomendaciones fundamentalmente en base a un sólo estudio que además de ser abierto fue detenido tempranamente por una desproporcionada RRR del $90 \%$ de eventos CV (Poldermans ${ }^{2}$ ) mientras que una vasta evidencia de BB en Insuficiencia Cardíaca muestran RRR de $15-35 \%^{3}$. Más recientemente las guías prácticas para cirugía no cardíaca del Colegio Americano de Cardiólogos establecieron que "todavía hay muy pocos ECCA sobre intervenciones médicas antes de cirugías no cardíacas ... y no proveen datos suficientes de los cuales extraer conclusiones y recomendaciones firmes ${ }^{4 "}$. Sin embargo, estas guías catalogaron como recomendación I el uso de BB en pacientes con isquemia detectada en el preoperatorio de cirugía vascular y recomendación lla en pacientes con hipertensión no tratada en el preoperatorio, enfermedad coronaria o factores de riesgo coronario mayores. El presente meta-análisis es metodológicamente correcto y aunque no exploró formalmente el sesgo de publicación la búsqueda fue exhaustiva e incluyó más estudios que las revisiones previas ${ }^{5}$. La heterogeneidad se observó en la eficacia (6 veces mayor en los ECCA de baja calidad) pero no en otros resultados como bradicar- dia e hipotensión, debilitando las conclusiones (de eficacia en este caso) que pudiera surgir de un meta-análisis. Además los metaanálisis están expuestos a sesgos como el de publicación* y a errores por azar por lo que los criterios de análisis debieran ser aún más estrictos que para un ECCA. Para que un meta-análisis pueda considerarse definitivo, sugiriendo no hacer futuros ECCA, debería cumplir al menos con los mismos criterios de suspensión de un estricto análisis interino* utilizados en los ECCA. Los autores del presente meta-análisis calcularon que se requerirían 6124 pacientes (asumiendo una tasa de eventos del 10\%) pero sólo se incluyó el $20 \%$ de esta muestra mínima. Un ECCA no podría detenerse en estas circunstancias y del mismo modo se requieren nuevas investigaciones para que la evidencia acumulativa del meta-análisis pudiera considerarse definitiva.

\section{Conclusiones del comentador}

Los BB perioperatorios posiblemente reduzcan el riesgo de eventos cardiovasculares pero aumentan el riesgo de bradicardia e hipotensión que requiere tratamiento. Considerando la insuficiente evidencia disponible es clara la necesidad de un gran ECCA que establezca definitivamente los beneficios y riesgos de los BB en cirugía no cardíaca. De hecho ha comenzado recientemente el estudio POISE que planea reclutar 10000 pacientes y ha reclutado a la fecha más de 4000 pacientes en 19 países entre los cuales participa la Argentina ${ }^{6}$. Deberemos aguardar sus resultados para aclarar definitivamente el tema y aplicar sus concluciones.

* ver glosario

\section{Dr. Agustín Ciapponi [ Unidad de Medicina Familiar y Preventiva. Hospital Italiano de Buenos Aires. ]}

Ciapponi A. Beta-bloqueantes en cirugía no cardíaca: evidencia promisoria pero insuficiente. Evid actual pract ambul 2005;8:136. Devereaux PJ, Beattie WS, Choi PT, Badner NH, Guyatt GH, et al. How strong is the evidence for the use of perioperative beta blockers in non-cardiac surgery? Systematic review and meta-analysis of randomised controlled trials. BMJ 2005;331(7512):313-21. PMID: 15996966

\section{Referencias}

1. Mangano DT. Perioperative cardiac morbidity. Anesthesiology 1990;72:153-84

2. Poldermans D, Boersma E, Bax JJ, Thomson IR, van de Ven LL, Blankensteijn JD et al. The effect of bisoprolol on perioperative mortality and myocardial infarction in high-risk patients undergoing vascular surgery. Dutch Echocardiographic Cardiac Risk Evaluation Applying Stress Echocardiography Study Group. N Engl J Med 1999;341[24]: 1789-94.

3. Yusuf S, Peto R, Lewis J, Collins R, Sleight P. Beta blockade during and after myocardial infarction: an overview of the randomized trials. Prog Cardiovasc Dis 1985;27: 335-71.

4. Eagle KA, Berger PB, Calkins H, Chaitman BR, Ewy GA, Fleischmann KE, et al. ACC/AHA guideline update for perioperative cardiovascular evaluation for noncardiac surgery - executive summary. A report of the American College of Cardiology / American Heart Association Task Force on Practice Guidelines (Committee to Update the 1996 Guidelines on Perioperative Cardiovascular Evaluation of Noncardiac Surgery). J Am Coll Cardiol 2002;39(3):542-53

5. Auerbach AD, Goldman L. Beta-blockers and reduction of cardiac events in noncardiac surgery: scientific review. JAMA 2002;287: 1435-44

6. Area de Investigación y Gestión Unidad de Medicina Familiar y Preventiva. Hospital Italiano de Buenos Aires. 